\title{
La Cour Nouvelle ou « Brûlée » - palais disparu des princes phanariotes de Valachie
}

The New or "Burnt" Court - Long Gone Palace of the Phanariot Princes of Wallachia

\section{Tudor Dinu}

\section{(2)enEdition}

\section{Journals}

Electronic version

URL: https://journals.openedition.org/ceb/4845

DOI: $10.4000 /$ ceb.4845

ISSN: 2261-4184

\section{Publisher}

INALCO

\section{Electronic reference}

Tudor Dinu, "La Cour Nouvelle ou « Brûlée » - palais disparu des princes phanariotes de Valachie", Cahiers balkaniques [Online], 42 | 2014, Online since 16 June 2014, connection on 07 July 2021. URL: http://journals.openedition.org/ceb/4845 ; DOI: https://doi.org/10.4000/ceb.4845

This text was automatically generated on 7 July 2021.

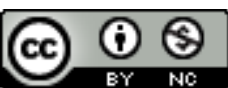

Cahiers balkaniques est mis à disposition selon les termes de la Licence Creative Commons Attribution - Pas d'Utilisation Commerciale 4.0 International. 


\section{La Cour Nouvelle ou « Brûlée » - palais disparu des princes phanariotes de Valachie}

The New or "Burnt" Court - Long Gone Palace of the Phanariot Princes of Wallachia

Tudor Dinu

1 À la suite des ravages produits par la guerre russo-turque de 1768-1774, Alexandre Ypsilanti, le nouveau prince régnant de Valachie, constata avec regret que la résidence de ses prédécesseurs connue sous le nom de Curtea Veche ("La Cour Ancienne ») était tellement délabrée qu'on ne pouvait plus la restaurer à des coûts acceptables. Un calcul estimatif lui montra que les travaux nécessaires pour la réfection du palais lui auraient coûté plus que la construction d'un nouvel édifice ${ }^{1}$. De nature pragmatique, le prince opta donc pour cette seconde solution, en démarrant les travaux de la «Nouvelle Cour » en mars 1774, quelques mois seulement après son arrivée à Bucarest.

\section{Une construction rapide}

Le lieu choisi pour la nouvelle résidence fut la plus haute butte des environs de la Capitale (Dealul Spirii), orientée vers la vallée de la rivière DâmboviŢa, sur laquelle elle offrait une belle perspective. Cette zone n'avait jamais été habitée et était couverte à l'époque par les vastes vignobles du couvent Mihai Vodă, l'un des plus importants producteurs de vin de la Valachie. Ainsi, elle présentait l'avantage de se trouver loin du tumulte du centre-ville, mais assez près de la capitale (environ deux kilomètres).

Pour Ypsilanti, qui logeait pour le moment dans les maisons des Brancovan situées sur la place aujourd'hui appelée PiaŢa Unirii², la construction du nouveau palais était une priorité pressante. Il confia donc la surveillance de l'ouvrage à quelques-uns des plus hauts dignitaires du pays, le grand ban Dimitrie Ghica, le velvornic Nicolae Dudescu et 
le medelnicer Ioan Vilara, dont le prince, qui n'était point avare quand il s'agissait de son propre confort, attendait au plus vite la fin des travaux.

Selon les conseils d'un ami intime du prince, le Suisse Franz-Joseph Sulzer, le projet de l'ensemble revint d'abord à un architecte de Braşov (Transylvanie), mais les plans présentés par celui-ci ne satisfirent pas les exigences des boyards. On recourut alors aux services d'un ingénieur italien, Spiridon Macri, qui « eut l'honneur de construire le grand escalier $»^{3}$ et la chapelle du palais ${ }^{4}$. La plupart des ouvriers et des contremaîtres étaient cependant des Roumains. En leur tenant la bride haute, les boyards les obligèrent à travailler hâtivement, avec des conséquences prévisibles sur la qualité de l'ouvrage. Un an et demi après le début des travaux, en novembre 1776, le palais proprement dit était achevé. Il ne restait qu'à parfaire le mur d'enceinte, les cuisines, les chambres des gardes et les écuries ${ }^{5}$. Pourtant, le chantier se prolongea jusqu'à la révocation du prince (1782).

Malgré les efforts soutenus fournis par les constructeurs, le résultat n'enthousiasma pas les visiteurs étrangers du palais d'Ypsilanti, même à l'époque de sa plus grande splendeur. Le susnommé Sulzer qui, outre son mépris souverain pour les Valaques et pour leurs mauvaises habitudes, aurait pu être soupçonné d'être aussi intéressé personnellement dans l'affaire, écrivait : «Ce palais est tout à fait irrégulier et pire que toute demeure de boyard »; « on a abouti [...], à grands frais, à un palais inhabitable à cause de la mauvaise odeur, et dont les couloirs sont dépourvus de lumière et même de marches ${ }^{6}$ (le distingué historien et musicien oublia, quelques lignes plus bas, cette dernière remarque, en parlant du grand escalier de l'édifice). D'ailleurs, cet élément d'architecture fut aussi le seul à attirer l'intérêt de l'aristocrate britannique Elisabeth Craven, reçue avec grande pompe à la cour de Nicolas Mavrogénis (« les marches d’un escalier imposant qui mène à la salle d'audience $»^{77}$.

6 Même les admirateurs de la résidence princière ne réussirent pas à attribuer au palais des qualités concrètes. L'ingénieur militaire Joseph Gabriel Monnier se contente d'une affirmation générale : "Le palais du prince ou hospodar est majestueux $»^{8}$, tandis que Denys Fotinos affirme laconiquement que "la Cour Princière [...] qui se trouve en amont du monastère Mihai Vodă est une merveille ${ }^{9}$ ». Pour le commerçant allemand Jenne (1786) l'édifice est au contraire « grand et laid » ${ }^{10}$.

7 Malheureusement, de telles opinions, manifestement subjectives, qu'elles soient favorables ou non, ne nous offrent aucune information positive sur la configuration concrète du palais d'Ypsilanti. Au surplus, la tâche de l'historien est rendue encore plus difficile par le sort défavorable de la Nouvelle Cour, qui tombera bientôt victime de deux incendies dévastateurs (1789 et 1812).

\section{Les preuves archéologiques}

8 Dans ces conditions, les informations les plus riches et crédibles sur le sujet proviennent des rapports archéologiques des fouilles effectuées au cours des années 1978-1981 à l'ancien emplacement du palais, où le chef du Parti communiste roumain avait prévu la construction du «centre politico-administratif de la patrie ${ }^{11}$. Dans des conditions de travail très dures et sous la pression des délais, l'équipe conduite par le chercheur Panait I. Panait, comprenant des archéologues et des professeurs de la Faculté d'Histoire de l'Université de Bucarest, a pu découvrir la plus grande partie des ruines du palais d'Ypsilanti ainsi que d'autres bâtiments du complexe 
voïvodal ${ }^{12}$. Cependant, étant donné la destruction à peu près complète subie plus d'un siècle auparavant par la Nouvelle Cour, les chercheurs n'ont pu identifier souvent que les fondations du palais et n'ont recueilli que quelques informations sur l'étage supérieur (piano nobile), où se trouvaient la salle du trône et les autres pièces officielles.

9 Avec sa surface de plus de $2000 \mathrm{~m}^{2}$, la résidence d'Ypsilanti était l'édifice le plus étendu construit à l'époque des phanariotes ${ }^{13}$. Elle se composait de deux grands bâtiments disposés en forme de L, dont l'un, orienté est-ouest, regardait vers la DâmboviŢa. À la différence de la plupart des résidences nobiliaires de la Valachie, le palais était bâti sur deux niveaux, rez-de-chaussée et étage, mais sans cave. C'est pourquoi on avait construit séparément d'imposants celliers en brique, destinés aux provisions solides et liquides de la Cour princière. Cet ensemble complexe de salles et de galeries couvertes de voûtes et de coupoles occupait au milieu du xxe siècle une surface de $310 \mathrm{~m}^{2}$ et offrait des conditions idéales pour la fermentation en bouteilles et pour le vieillissement des boissons mousseuses, de telle manière qu'il servira successivement à l'entreprise de Wilhelm Mott ${ }^{14}$, fondée en 1912 et, après la nationalisation de 1948, à la firme communiste Zarea.

10 L'aile orientée est-ouest était longue de $45 \mathrm{~m}$ et large de $23 \mathrm{~m}$. Elle se composait d'une grande salle centrale et de plusieurs pièces, de formes et de surfaces variables, situées sur ses deux côtés. Outre les 21 salles identifiées par les archéologues, le bâtiment comprenait encore cinq tours avancées, dont quatre vers la Dâmboviţa et une vers la cour intérieure. Sensiblement plus modeste, l'autre corps de bâtiment occupait une surface de seulement $690 \mathrm{~m}^{2}$ et ne comprenait que 11 pièces. Les matériaux de construction employés pour le rez-de-chaussée du palais étaient tout à fait banals : la brique couverte d'un crépi jaune pour les murs, des poutres en bois pour le plafond et de la terre compactée pour le plancher. Cette austérité était due au fait que ces pièces étaient destinées aux services administratifs ( et au niveau inférieur se trouvaient les autorités : la trésorerie, les logothètes, l'état-major de l'armée et d'autres avec leurs bureaux $»^{15}$ ).

11 En échange, il est bien possible que les chambres de l'étage, réservées au prince et à sa famille, aient été dallées de marbre et les encadrements des fenêtres sculptés en pierre $^{16}$. Quoi qu'il en soit, les témoignages des visiteurs indiquent la présence, même en haut, d'une décoration très sobre, voire pauvre ( «la chambre d'audience [d'Alexandre Mourousis] est spacieuse, mais blanchie à la chaux: la multitude des fenêtres lui conférait un air joyeux $\|^{17}$; " cette salle blanchie à la chaux [le salon de la princesse Ypsilanti, n. n.], dont les fenêtres étaient ornées d'indienne rouge, n'avait comme seule décoration que quelques miroirs ordinaires $\left.»^{18}\right)$. Pourtant, divers objets découverts pendant les fouilles (vases de verre incrustés d'or, petites tasses à café de provenance occidentale, verreries de Murano ${ }^{19}$ ) mettent en lumière le luxe de la cour des phanariotes.

12 Hormis le palais proprement dit, les archéologues ont déterré aussi la chapelle princière consacrée à Sainte Catherine et divers bâtiments annexes. Mais la réalisation la plus importante des chercheurs a été la mise en évidence des dimensions impressionnantes de l'ensemble palatial ${ }^{20}$ (plus de $50000 \mathrm{~m}^{2}$ ), qui se présentait comme une ville indépendante, capable de s'assurer seule les ressources nécessaires à sa subsistance. Ce vaste espace comprenait deux cours fortifiées. Dans la cour extérieure se trouvaient les baraques des gardes et les dépendances de la résidence princière, tandis que la cour intermédiaire contenait le palais, l'église et les kiosques. Cet aspect 
un peu bizarre surprenait les voyageurs étrangers, habitués aux résidences somptueuses de l'Europe occidentale. C'est le cas par exemple de Christine Reinhard qui arrivée à Bucarest en 1806 et bien qu'avertie sur " le train de vie simple du palais ", n'a pu cacher sa surprise d'être obligée, pour rendre visite au prince, de traverser une basse-cour pleine de bestiaux et de volailles ${ }^{21}$.

Malgré les espoirs d'Ypsilanti, son établissement sera malchanceux. Sept ans seulement après sa révocation (qui eut lieu le 14/25 décembre 1789), son palais fut incendié par les troupes autrichiennes libératrices, venue pour civiliser les barbares valaques ${ }^{22}$. Ces derniers étaient si rétrogrades qu'ils s'opposaient les armes à la main au recyclage des matériaux de construction de la Nouvelle Cour, interdisant aux Autrichiens « de voler le bois et le fer des bâtiments et de détruire la clôture de la cour " ${ }^{23}$. Cependant, le modèle occidental a été adopté par beaucoup d'indigènes, de telle manière qu'à la fin de la guerre russo-turque de 1787-1791 le palais était devenu inhabitable et le pouvoir s'est vu obligé de déménager en demandant asile aux monastères Mihai Vodă et Saint Sabba (Sfântul Sava).

Cette situation a paru intolérable au prince Constantin Hangerli, qui, à peine arrivé sur les rives de la DâmboviŢa, se mit en quatre pour restaurer la cour : il vendit aux enchères le terrain de l'ancienne résidence princière, augmenta les impôts, instaura un régime de terreur parmi ceux qui participaient à la reconstruction du palais. Tout le pays travailla pendant plusieurs mois pour satisfaire les désirs du maître ${ }^{24}$.

Puisqu'on avait besoin d'ouvriers (maçons, charpentiers...) et que les étrangers ne répondaient pas à l'appel du prince, Constantin Hangerli ordonna qu'on amène de force tous ceux qui travaillaient à d'autres constructions de la capitale. De plus, « les Tsiganes qui savaient travailler le fer pour les portes et les fenêtres » et qui se trouvaient en ville furent contraints de se présenter d'urgence à la cour, sans qu'il fût tenu compte de ceux auxquels ils appartenaient et des raisons de leur présence. Théoriquement, tous les ouvriers devaient être rémunérés, mais le prince pensa qu'il serait plus rationnel d'ajourner le paiement des salaires jusqu'à la fin des travaux, sous le prétexte que les ouvriers ne se tuaient pas à la tâche. Quant aux Tsiganes manouvriers qui appartenaient aux couvents Văcărești, Cotroceni, Radu Vodă de Bucarest et Glavacioc du district d'Argeş, ils ne recevaient en tout cas aucun salaire, car l'argent qui leur revenait entrait de droit dans les trésoreries des monastères respectifs. De toute manière, Hangerli n'avait pas été très généreux envers les moines, ce qui détermina le supérieur du couvent de Cotroceni à s'esquiver en n'envoyant pas au palais tous les Tsiganes du monastère. Quand le prince découvrit la ruse de l'abbé, il ordonna la confiscation de tous les serfs non déclarés. Après cette expérience, personne ne refusa plus les demandes du souverain. À la cour arrivaient sans cesse des chariots chargés de provisions, outils de travail, matériaux de construction (briques, bois scié, chaux, sable, etc.) et les ouvriers travaillaient sans relâche, mais sans réussir à achever l'œuvre au terme établi par le prince. Alors, le 3 octobre 1798, fâché parce que l'on avait «troublé [s]on altesse, quand il était bien le temps pour déménager", Hangerli ordonna sévèrement "qu'on achève les travaux la semaine prochaine ${ }^{25}$, les coupables étant, dans le cas contraire, passibles du bagne. Personne n'étant attiré par cette seconde variante, le prince vit son rêve accompli sur-le-champ et put changer immédiatement de domicile. Cour était, de l'avis des archéologues, assez modeste. Elle prouvait «le déclin des 
techniques de construction et des matériaux utilisés $»^{26}$. Au rez-de-chaussée, les seules interventions notables datant de cette période sont la création d'une vaste salle $\left(132 \mathrm{~m}^{2}\right)$ et le remplacement du plancher en terre battue par un pavement de briques. Outre cela, cette même année (1798), on a construit des nouveaux poêles ornés de plaques de faïence à motifs floraux.

Le confort relativement réduit du palais n'empêchait pas le prince d'y donner des banquets opulents, comme celui occasionné par la visite à Bucarest du commandant de la flotte ottomane Küçük Hussein avec sa suite. L'hospitalier amphitryon a eu alors l'obligeance de consoler ses hôtes qui n'avaient pu apporter avec eux leurs harems. La tâche n'était pas facile puisque les dignitaires turcs ne voulaient pas se contenter de courtisanes ordinaires, mais prétendaient avoir accès aux charmes des femmes des plus hauts boyards. Et étant donné que ceux-ci refusèrent fermement de partager leurs femmes avec les païens, l'ingénieux prince «envoya dans les rues le maréchal de la cour et les intendants pour apporter des putains, choisies parmi les plus belles, les vêtir avec des tenues provenant de la garde-robe princière, et leur promettre de riches récompenses afin qu'elles satisfassent tous les goûts du pacha et de ses compagnons ". Le succès de l'opération fut total : les Turcs firent la noce jusqu'au jour avec les fausses femmes de boyards et leur donnèrent même des cadeaux ${ }^{27}$ pour leur aimable prestation. Hangerli s'assura ainsi la bienveillance des potentats ottomans, mais pas pour longtemps, car il eut l'inspiration malheureuse de comploter justement contre le pacha Hussein et ses machinations arrivèrent à la connaissance du tout puissant commandant de la flotte.

18 Naturellement survint sa révocation ( 31 janvier 1799), mais le prince était encore optimiste, parce qu'il avait écrit à son ancien protecteur qui lui promit «de faire ce qu'il pouvait " "pour obtenir une nouvelle nomination, cette fois-ci comme prince régnant de la Moldavie $»^{28}$. Hangerli attendait donc tranquillement le firman du sultan, avec le seul regret de devoir abandonner le palais qu'il venait justement de restaurer. Mais le 18 février survint le coup de théâtre: le peşkirgi Hussein accompagné d'un nègre " terrible, grand, lippu, noir, immonde $»^{29}$ et de deux serviteurs franchit le seuil de la cour princière. Hanté par un pressentiment, Hangerli hésita à recevoir les messagers de la Sublime Porte. Invoquant son état de santé, il obtint un court délai " pour déguster [s] on sorbet », car il avait « mal à la poitrine $»^{30}$. Après avoir repris son courage, il les invita " dans la salle du trône en présence des boyards » ${ }^{31}$, où il leur offrit des chibouks et des cafés. Le peşkirgi Hussein rivalisait de louanges orientales et donnait au prince des garanties qu'il allait être nommé sur le trône moldave, mais Hangerli était de plus en plus inquiet en raison de l'aspect féroce du Nègre, qu'il voulut même évacuer de la pièce, initiative qui se solda par une réponse tranchante: "istemem, c'est-à-dire je ne veux pas $\|^{32}$. La situation devenait critique. Le prince demanda en grec au porte-épée Mavrocordat d'appeler les gardes, mais, hélas, l'assassin était hellénophone. Sans perdre de temps, celui-ci jeta vers lui un lacet qui rata sa cible. Alors, il ôta deux pistolets qu'il déchargea dans la poitrine de Hangerli (" puisque le Nègre avait / deux révolvers cachés »3). Puis, avec un kandjar, il mit en pièces sa victime et finit par la décapiter pour envoyer sa tête comme trophée à la Sublime Porte. L'assassin ne manqua pas l'occasion de s'approprier «l'argent, la montre et les bagues $\|^{34} \mathrm{du}$ prince, avant de déshabiller son cadavre et de le traîner dans la cour pour l'exposer à l'opprobre de ses anciens sujets. Il y avait là des gens disposés à profaner son corps ( « un gredin, accablé par le fardeau des impôts, jeta un sou sur la dépouille en disant : "sois rassasié, monstre cupide !" ${ }^{35}$ ). Les bardes valaques 
furent sincèrement impressionnés par la fin violente du prince étranger et ils l'ont transformé en un héros tragique en lui dédiant des ballades pleines d'enseignements moraux.

\section{Les catastrophes}

19 Les bourreaux turcs, eux, ne se contentèrent pas de profaner le cadavre, mais " commencèrent à piller le palais », confisquèrent " en faveur de l'empire » « les biens des domestiques, des parents du prince et de tous ceux qui logeaient à la cour $»^{36}$ et soumirent à la torture tous les proches de Hangerli suspectés d'avoir caché certaines richesses du défunt. Et, comme si tous ces crimes n'étaient pas suffisants, deux ans après, la Nouvelle Cour fut dévastée encore une fois au moment où le prince Michel Soutzo, ses plus importants boyards et une bonne partie de la population abandonnèrent la Capitale à cause des rumeurs au sujet d'une invasion imminente des troupes de Pazvantoglu, le pacha rebelle de Vidin. Cette fois, les auteurs du pillage étaient « des gens désespérés et sans métier que le peuple appelait crai (libertins)». Ceux-ci profitèrent de la dissolution de l'autorité pour pénétrer dans la cour princière « et voler tout ce qui s'y trouvait $»^{37}$ (« les crai transportaient les outils, les armes, les litières, l'argenterie, les registres de la trésorerie, absolument tout ${ }^{38}$ ). Ils prirent même les insignes du pouvoir, « les tuiuri et les étendards », et ils « défilaient dans les rues conduits par leur chef de bande, le camelot Melamos, qui portait sur sa tête le bonnet de cérémonie du prince $\aleph^{39}$ ). Il ne s'agissait cependant pas d'une promenade innocente, car «ils brisaient les portes des tavernes et des caves, y entraient et s'adonnaient aux plus répugnantes débauches $»^{40}$.

20 "L'épouvantable tremblement de terre " survenu le 14 octobre 1802, qui obligea le nouveau prince régnant Constantin Ypsilanti à s'installer provisoirement au monastère Cotroceni ${ }^{41}$, aggrava encore l'état déjà déplorable de la Nouvelle Cour. Rentré après quelques réparations superficielles, celui-ci manifesta un intérêt particulier pour les fortifications ${ }^{42}$, mais l'aspect du palais resta minable ( les salons de l'entrée étaient sales, sombres [...] le pavage - détérioré », écrivait la voyageuse allemande Christine Reinhard ${ }^{43}$ ). Pourtant, faute de mieux, l'édifice continua d'offrir abri au prince et à l'administration militaire pendant la guerre russo-turque de 1806-1812. Cette même guerre empêcha une restauration radicale de la Nouvelle Cour, tâche qui devait revenir au prince Jean Georges Caradja, arrivé à Bucarest le 12 décembre $1812^{44}$.

21 Mais juste avant le démarrage des travaux, à l'aube du 22 décembre ${ }^{45}$, un incendie terrible dévora jusqu'aux fondations tous les bâtiments du complexe. Le sinistre a été décrit par le comte Auguste de Lagarde qui se trouvait alors à Bucarest : «À cinq heures du matin, un bruit assourdissant me réveilla à l'improviste. J'ai cru que la ville était saccagée ; dans la chambre régnait une lumière extraordinaire. J'ai couru à la fenêtre et j'ai vu, juste devant moi, le palais du prince de la Valachie enveloppé de flammes. Situé sur une colline, il évoquait le Vésuve dans toute sa violence; les langues de feu menaçaient d'incendier toute la ville. Bâti en bois, presque en totalité, Bucarest risquait de prendre feu de tous côtés. Je me suis habillé hâtivement et j'ai couru pour regarder de plus près cette calamité. Les mesures de sauvetage ont été prises sans délai et elles furent bien conçues. Des pompiers portant une sorte de casque et un habit romain montaient sans peur sur les toits ardents et entraient dans les pièces embrasées pour sauver quelques meubles; mais, malgré leurs efforts, le feu se propageait rapidement. 
Tous les habitants étaient debout ; il faisait jour comme à midi $»^{46}$. En effet, rien n'a pu être sauvé du désastre, le prince perdant lui-même des biens estimés à 300000 piastres.

Cependant la vie continuait, aspect dont notre comte était tout à fait conscient. Il profita donc des événements pour flirter avec la jeune sœur du préfet de police Grigore Filipescu à laquelle il offrit poliment le bras pour une promenade à la lumière des flammes, suivie d'un petit déjeuner romantique ${ }^{47}$. De toute façon, la Nouvelle Cour ne pouvait plus être sauvée, quoique, selon les constatations des archéologues, on y a encore entrepris des travaux de faible ampleur et de qualité douteuse (réfection de quelques murs avec des briques pas assez cuites, pavage de certaines pièces avec une couche de glaise mélangée de fragments de brique ${ }^{48}$ ) afin d'abriter dans l'ancien palais une partie des bureaux administratifs. Mais, étant donné que les dépenses nécessaires pour les travaux de réparation et de maintenance dépassaient sensiblement le budget de la Cour, l'édifice fut laissé en ruines; le prince déménagea pour de bon et s'installa dans une résidence plus modeste.

\section{BIBLIOGRAPHY}

Busuioceanu, Alexandru (1929), Un palat domnesc din vremea fanarioţilor : Curtea Nouă din Bucureşti, Buletinul Comisiunii Monumentelor Istorice (XXII), 1929.

Cantea Gheorghe ( =Cantacuzino), (1959), Cercetări arheologice pe dealul Mihai Vodă şi împrejurimi, în Bucureștii de odinioară în lumina săpăturilor arheologice, sous la rédaction de I. Ionaşcu, Bucureşti, Editura ştiinţiifică.

Dionisie, Fotino, (1818) Istoria Generală a Daciei, Vienne.

Eclesiarhul Dionisie, (1934) Cronograful Ţării Româneşti, avec une préface de Dr. C. S. NicolăescuPlopşor, Tipografia „Episcopul Vartolomeiu” Râmnicul-Vâlcea.

Giurescu Constantin (1926), Uciderea voievodului Constantin Hangerli - o povestire în versuri necunoscută, dans Revista istorică, XII (10-12).

Hurmuzaki, Eudoxiu de, Documente privitoare la istoria românilor culese de, vol. XIX, part I.

Panait, Panait Ion, (1980), Cercetări privind palatul CurŢii Noi din Bucureşti, în Materiale şi cercetări arheologice, A XIV-a Sesiune de Rapoarte Arheologice, Tulcea.

Panait, Panait Ion, (1981), Cercetări arheologice la Palatul domnesc de la Curtea Nouă din Bucureşti, în Materiale şi cercetări arheologice, a XV-a Sesiunea de Rapoarte Arheologice, Braşov, pp. 537-540.

Panait, Panait Ion, (1979), Raport preliminar asupra cercetărilor arheologice dela Curtea Nouă din București, în Materiale şi cercetări arheologice, A XIII-a Sesiune de Rapoarte Arheologice, Oradea. Românul Zilot, (1884), Ultima Cronică Română din Epoca Fanarioţilor, București, p. 13. 


\section{NOTES}

1. Panait I. Panait, Aristide ştefănescu, op. cit., p. 68.

2. Al. Busuioceanu, Un palat domnesc din vremea fanarioţilor: Curtea Nouă din Bucureşti, Buletinul Comisiunii Monumentelor Istorice (XXII), 1929, p. 123.

3. Călători străini despre Țările Române, vol. x, partea I, op. cit, pp. 462-463.

4. Stoicescu, Repertoriul bibliografic..., op. cit., p. 36.

5. Hurmuzaki, Eudoxiu de, Documente privitoare la istoria românilor culese de, vol. XIV, partea II, pp. 1231-1232.

6. Călători străini despre Ţările Române, vol. x, partea I, op. cit., p. 462.

7. Ibidem, p. 721.

8. Ibid., p. 787.

9. Istoria Generală a Daciei sau a Transilvaniei, Ţării Munteneşti şi a Moldovei, traducere din greceşte de George Sion, Editura Valahia, 2008, p. 314.

10. Stoicescu, Repertoriul bibliografic..., op. cit., p. 36.

11. Panait. I. Panait, Raport preliminar asupra cercetărilor arheologice dela Curtea Nouă din București, în Materiale şi cercetări arheologice, A XIII- $a$ Sesiune de Rapoarte Arheologice, Oradea, 1979, p. 396.

12. Ibidem, pp.395-398 ; Panait. I. Panait, Cercetări privind palatul CurŢii Noi din Bucureşti, în Materiale şi cercetări arheologice, A XIV-a Sesiune de Rapoarte Arheologice, Tulcea, 1980, pp. 632-635, Panait. I. Panait, Cercetări arheologice la Palatul domnesc de la Curtea Nouă din Bucureşti, în Materiale şi cercetări arheologice, a XV-a Sesiunea de Rapoarte Arheologice, Braşov, 1981, pp. 537-540.

13. Les recherches archéologiques de l'année 1980 ont découvert $2.040 \mathrm{~m}^{2}$ (cf. Panait. I. Panait, Cercetări arheologice la Palatul domnesc de la Curtea Nouă, op. cit. p. 539).

14. Gheorghe Cantea (=Cantacuzino), Cercetări arheologice pe dealul Mihai Vodă şi împrejurimi, în Bucureştii de odinioară în lumina săpăturilor arheologice, sous la rédaction de I. Ionaşcu, Bucureşti, Editura ştiinŢiifică, 1959, p. 119.

15. Stoicescu, Repertoriul bibliografic..., op. cit. p. 37.

16. Panait. I. Panait, Cercetări arheologice la Palatul domnesc de la Curtea Nouă din București, op. cit., p. 539.

17. Vince Batthyány dans Călători străini despre Ţările Române în secolul al XIX-lea, op.cit., vol. I, p. 91.

18. Christine Reinhard dans Călători străini despre Ţările Române în secolul al XIX-lea, op. cit., vol. I, p. 288.

19. Panait. I. Panait, Cercetări arheologice la Palatul domnesc de la Curtea Nouă din București, op. cit., p. 539.

20. Panait. I. Panait, Raport preliminar asupra cercetărilor arheologice de la Curtea Nouă din Bucureşti, op. cit., p. 397.

21. Călători străini despre Ţările Române în secolul al XIX-lea, op. cit., vol. I, p. 287.

22. Hurmuzaki, Eudoxiu de, Documente privitoare la istoria românilor culese de, vol. XIX, part I, p. 571.

23. Stoicescu, Repertoriul bibliografic..., op. cit. p. 37, v. A. Urechia, Istoria Românilor, op. cit., vol III, p. 364.

24. Tous les documents cités ci-dessous se trouvent dans V. A. Urechia, Istoria Românilor, op. cit., vol viI, p. 223-226.

25. Ibidem, p. 225.

26. Panait. I. Panait, Cercetări privind palatul CurŢii Noi din Bucureşti, op. cit., p. 634.

27. Dionisie Eclesiarhul, Cronograful Ţării Românești, avec une préface de Dr. C.S. NicolăescuPlopşor, Tipografia „Episcopul Vartolomeiu” Râmnicul-Vâlcea, 1934, p. 96. 
28. Dionisie Fotino, Istoria Generală a Daciei ..., op. cit. p. 330.

29. Stihuri asupra venirii nemţilor în Bucureşti şi asupra pieirii voievodului Hangerli de un anonym, edité par Gregoriu Gr. Tocilescu dans Revista pentru istorie, archeologie şi filologie, an I, volume I, fasc. II, 1883, p. 330.

30. C. C. Giurescu, Uciderea voievodului Constantin Hangerli - o povestire în versuri necunoscută, dans Revista istorică, XII (10-12), 1926, p. 325.

31. Stihuri asupra venirii nemŢilor în București şi asupra pieirii voievodului Hangerli de un anonym, op. cit., p. 329.

32. Dionisie Fotino, Istoria Generală a Daciei ..., op. cit., p. 331.

33. Stihuri asupra venirii nemŢilor în Bucureşti şi asupra pieirii voievodului Hangerli de un anonym, op. cit., p. 330.

34. Dionisie Eclesiarhul, Cronograful Ţării Românești, op. cit, p. 104.

35. Zilot Românul, Ultima Cronică Română din Epoca Fanarioţilor, Bucureşti, 1884, p. 13.

36. Dionisie Fotino, Istoria Generală a Daciei ..., op. cit., p. 333.

37. Ibidem, p. 346.

38. Cronica meşteşugarului Ioan Dobrescu (1802-1830), édition Ilie Corfus, dans Studii şi articole de istorie, VIII (1968), p. 321.

39. Cronica meşteşugarului Ioan Dobrescu, op. cit., p. 321-322.

40. Dionisie Fotino, Istoria Generală a Daciei ..., op. cit., p. 347.

41. Ibidem, p. 353.

42. Naum Râmniceanu dans Constantin Erbiceanu, Cronicari greci, op. cit., p. 264.

43. Călători străini despre Ţările Române în secolul al XIX-lea, op. cit., vol. I, p. 288.

44. Dionisie Fotino, Istoria Generală a Daciei ..., op. cit., p. 398.

45. Cronica meşteşugarului Ioan Dobrescu, op. cit., p. 337.

46. Călători străini despre Ţările Române în secolul al XIX-lea, op. cit., vol. I, p. 563.

47. Ibidem, p. 563-564.

48. Panait. I. Panait, Cercetări privind palatul CurŢii Noi din Bucureşti, op. cit., p. 634.

\section{ABSTRACTS}

In our present paper we have attempted to recreate the image of the palace erected by prince Alexander Ipsilanti (1774-1782) on the Spirea Hill nearby the capital, resorting to the available written sources (mainly testimonies of the foreign travellers) and the results of the archaeological excavations carried out between 1978 and 1981. We have also followed the turbulent history of the princely residence severely damaged by two great fires in 1789 and 1812 and one earthquake in 1802, showing interest in the restoration works realised during the reign of Constantine Hangerli (1797-1799) and Constantine Ipsilanti (1802-1806). Finally, we have related some memorable episodes hosted in the court, such as the great banquet offered in 1798 in honour of the commander of the Ottoman fleet Küçük Husein and the ferocious murder of prince Hangerli, one year later.

Parmi les résidences bucarestoises des princes régnants de Valachie, le palais bâti par Alexandre Ypsilanti (1774 - 1782) sur la colline dite « de Spirea » (Dealul Spirii) est l'une des moins connues aujourd'hui, étant donnée sa très courte période d'existence (les incendies de 1789 et 1812 ainsi 
que le tremblement de terre de 1802 l'avaient détruit presque entièrement, il y a déjà deux siècles). En partant des sources écrites disponibles et des résultats des fouilles archéologiques entreprises au cours des années 1978-1981, nous avons essayé de reconstituer l'aspect originel de cette cour princière et de retracer l'histoire des changements qu'elle a subis au temps de Constantin Hangerli (1797-1799) et de Constantin Ypsilanti (1802-1806). Sont évoqués aussi quelques épisodes mémorables qui s'y sont déroulés, par exemple le grand banquet offert en 1798 à l'amiral-en-chef de la flotte ottomane Küçük Hussein ou l'assassinat spectaculaire du prince Hangerli, l'année suivante.

\section{INDEX}

Subjects: Histoire

Mots-clés: Ypsilanti Alexandre (1774-1782), Ypsilanti Alexandre (1725-1807), Phanariotes, Hangerli Constantin (1797-1799), Hangerli Constantin (1797-1799), Ypsilanti Constantin (1760-1816), Ypsilanti Constantin (1802-1806)

Keywords: Ypsilanti Alexander (1725-1807) Phanariots, Hangerli Constantine (1760-1799), Ypsilanti Constantin (1760-1816), Bucharest, Eighteenth century, History, Architecture Geographical index: Bucarest motsclestr Ypsilanti Alexander (1725-1807), Fenerliler, Hangerli Constantinos (1760-1799), Ypsilanti Constantinos (1760-1816) Bükreş, On sekizinci yüzyıllarda, Tarih, Mimarlık motsclesmk УПСИЛАНТИС АЛЕКСАНДАР (1725-1807), ФЕНЕР, ХАНГЕРЛИ КОНСТАНТИН (1760-1799), УПСИЛАНТИС КОНСТАНТИН (1760-1816), БУКУРЕШТ, ОСУМНАЕСЕТТИОТ ВЕК, ИСТОРИЈА, АРХИТЕКТУРА

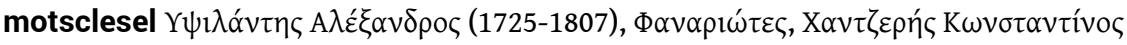

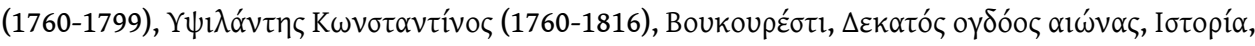

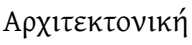

Chronological index: dix-huitième siècle

\section{AUTHOR}

\section{TUDOR DINU}

Université de Bucarest 